\title{
Erratum to: Diagnosis and Evaluation
}

\author{
Flávio Danni Fuchs
}

Erratum to: Chapter 3 in: F.D. Fuchs, Essentials of Hypertension, https://doi.org/10.1007/978-3-319-63272-8_3

1) The following text was removed from the last paragraph of Section 3.4.1.

The value proposed for systolic BP during sleep $(100 \mathrm{mmHg})$ exceeds the expected $10 \%$ dipping of $\mathrm{BP}$ in relation to the value proposed for daily $\mathrm{BP}$, and is rarely seen in ABP exams in the daily practice. Probably these values will be discussed by other scientific societies, but in general the values of the table are those expected for the diagnosis of hypertension with BP measured out of office.

2) The following values in table 3.2 were changed.

Daytime BP: $\geq 120 / 80$ by $\geq 130 / 80$ for both ABP and HBP

Nighttime BP: $\geq 100 / 65$ by $\geq 110 / 65$

24-h BP: $\geq 115 / 75$ by $125 / 75$

The updated online version of this chapter can be found at https://doi.org/10.1007/978-3-319-63272-8_3 